\title{
The Cosmological Constant Problem and Nonlocal Quantum Gravity
}

\author{
J. W. Moffat \\ Department of Physics, University of Toronto, Toronto, Ontario M5S 1A\%, \\ Canada \\ and \\ Perimeter Institute for Theoretical Physics, Waterloo, Ontario N2J 2W9, \\ Canada
}

\begin{abstract}
A nonlocal quantum gravity theory is presented which is finite and unitary to all orders of perturbation theory. Vertex form factors in Feynman diagrams involving gravitons suppress graviton and matter vacuum fluctuation loops by introducing a low-energy gravitational scale, $\Lambda_{\text {Gvac }}<2.4 \times 10^{-3} \mathrm{eV}$. Gravitons coupled to non-vacuum matter loops and matter tree graphs are controlled by a vertex form factor with the energy scale, $\Lambda_{G M}<1-10 \mathrm{TeV}$. A satellite Eötvös experiment is proposed to test a violation of the equivalence principle for coupling of gravitons to pure vacuum energy compared to matter.
\end{abstract}

Talk given at the MRST 2002 Meeting, Perimeter Institute for Theoretical Physics, May 1-17, 2002, Waterloo, Ontario, Canada, and at the XVIIIth IAP Colloquium: Observational and Theoretical Results on the Accelerating Universe, July 1-5, 2002, Paris, France.

Dedicated to the memory of George Leibbrandt.

e-mail: john.moffat@utoronto.ca 


\section{Introduction}

It is generally agreed that the cosmological constant problem is one of the most severe problems facing modern particle and gravitational physics. It is believed that its solution could significantly alter our understanding of particle physics and cosmology [1].

There is now mounting observational evidence [2] that the universe is accelerating and that there exists some form of dark energy. One possible explanation for the accelerating expansion of the universe is a small cosmological constant corresponding to a vacuum energy density, $\rho_{\text {vac }} \sim\left(2.4 \times 10^{-3} \mathrm{eV}\right)^{4}$.

There have been many attempts to solve the cosmological constant problem (CCP). Weinberg's theorem [3] disallows all adjustment models involving extra fields such as a dynamical scalar field. Higher-dimensional models of the brane-bulk type with finite volume extra-dimensions do not avoid finetuning 近.

Superstring theory (M-theory) has not yet provided a solution to the CCP. This could be due to the problem of understanding how to introduce supersymmetry breaking into string theory models, although there may be some deeper reason for the failure.

In the following, I will describe a possible resolution of the CCP, based on a model of a nonlocal quantum gravity theory and field theory that suppresses the coupling of gravity to vacuum energy density. The theory can be tested by performing Eötvös experiments on Casimir vacuum energy in satellites.

\section{Gravitational Coupling to Vacuum Energy}

We can define an effective cosmological constant

$$
\lambda_{\text {eff }}=\lambda_{0}+\lambda_{\text {vac }}
$$

where $\lambda_{0}$ is the "bare" cosmological constant in Einstein's classical field equations, and $\lambda_{\text {vac }}$ is the contribution that arises from the vacuum density $\lambda_{\mathrm{vac}}=8 \pi G \rho_{\mathrm{vac}}$.

Already at the standard model electroweak scale $\sim 10^{2} \mathrm{GeV}$, a calculation

of the vacuum density $\rho_{\mathrm{vac}}$, based on local quantum field theory, results in a discrepancy of order $10^{55}$ with the observational bound

$$
\rho_{\mathrm{vac}} \leq 10^{-47}(\mathrm{GeV})^{4} \text {. }
$$


This results in a severe fine-tuning problem of order $10^{55}$, since the virtual quantum fluctuations giving rise to $\lambda_{\text {vac }}$ must cancel $\lambda_{0}$ to an unbelievable degree of accuracy. This is the "particle physics" source of the cosmological constant problem.

\section{$3 \quad$ Nonlocal Quantum Gravity}

Let us consider a model of nonlocal gravity with the action $S=S_{g}+S_{M}$, where $\left(\kappa^{2}=32 \pi G\right)$ 円:

$$
S_{g}=-\frac{2}{\kappa^{2}} \int d^{4} x \sqrt{-g}\left\{R\left[g, \mathcal{G}^{-1}\right]+2 \lambda_{0}\right\}
$$

and $S_{M}$ is the matter action, which for the simple case of a scalar field $\phi$ is given by

$$
S_{M}=\frac{1}{2} \int d^{4} x \sqrt{-g}\left(g^{\mu \nu} \mathcal{G}^{-1} \nabla_{\mu} \phi \mathcal{F}^{-1} \nabla_{\nu} \phi-m^{2} \phi \mathcal{F}^{-1} \phi\right) .
$$

Here, $\mathcal{G}$ and $\mathcal{F}$ are nonlocal regularizing, entire functions and $\nabla_{\mu}$ is the covariant derivative with respect to the metric $g_{\mu \nu}$. As an example, we can choose the covariant functions

$$
\begin{gathered}
\mathcal{G}(x)=\exp \left[-\mathcal{D}(x) / \Lambda_{G}^{2}\right], \\
\left.\mathcal{F}(x)=\exp \left[-\left(\mathcal{D}(x)+m^{2}\right) / \Lambda_{M}^{2}\right)\right],
\end{gathered}
$$

where $\mathcal{D} \equiv \nabla_{\mu} \nabla^{\mu}$, and $\Lambda_{G}$ and $\Lambda_{M}$ are gravitational and matter energy scales, respectively [5, 6].

We expand $g_{\mu \nu}$ about flat Minkowski spacetime: $g_{\mu \nu}=\eta_{\mu \nu}+\kappa h_{\mu \nu}$. The propagators for the graviton and the $\phi$ field in a fixed gauge are given by

$$
\bar{D}^{\phi}(p)=\frac{\mathcal{G}(p) \mathcal{F}(p)}{p^{2}-\bar{m}^{2}+i \epsilon},
$$

\footnotetext{
${ }^{1}$ The present version of a nonlocal quantum gravity and field theory model differs in detail from earlier published work [5, 6. A paper is in preparation in which more complete details of the model will be provided.
} 


$$
\bar{D}_{\mu \nu \rho \sigma}^{G}(p)=\frac{\left(\eta_{\mu \rho} \eta_{\nu \sigma}+\eta_{\mu \sigma} \eta_{\nu \rho}-\eta_{\mu \nu} \eta_{\rho \sigma}\right) \mathcal{G}(p)}{p^{2}+i \epsilon}
$$

where $\bar{m}^{2}=m^{2} \mathcal{G}(p)$.

Unitarity is maintained for the S-matrix, because $\mathcal{G}$ and $\mathcal{F}$ are entire functions of $p^{2}$, preserving the Cutkosky rules.

Gauge invariance can be maintained by satisfying certain constraint equations for $\mathcal{G}$ and $\mathcal{F}$ in every order of perturbation theory. This guarantees that $\nabla_{\nu} T^{\mu \nu}=0$.

\section{Resolution of the CCP}

In flat Minkowski spacetime, the sum of all disconnected vacuum diagrams $C=\sum_{n} M_{n}^{(0)}$ is a constant factor in the scattering S-matrix $S^{\prime}=S C$. Since the S-matrix is unitary $\left|S^{\prime}\right|^{2}=1$, then we must conclude that $|C|^{2}=1$, and all the disconnected vacuum graphs can be ignored. This result is also known to follow from the Wick ordering of the field operators.

Due to the equivalence principle gravity couples to all forms of energy, including the vacuum energy density $\rho_{\text {vac }}$, so we can no longer ignore these virtual quantum fluctuations in the presence of a non-zero gravitational field. Quantum corrections to $\lambda_{0}$ come from loops formed from massive standard model (SM) states, coupled to external graviton lines at essentially zero momentum.

Consider the dominant contributions to the vacuum density arising from the graviton-standard model loop corrections. We shall adopt a model consisting of a photon loop coupled to gravitons, which will contribute to the vacuum polarization loop coorection to the bare cosmological constant $\lambda_{0}$. The covariant photon action is [7]:

$$
S_{A}=-\frac{1}{4} \sqrt{-g} g^{\mu \nu} g^{\alpha \beta} \mathcal{G}^{-1} F_{\mu \alpha} \mathcal{F}^{-1} F_{\nu \beta}
$$

with

$$
F_{\mu \alpha}=\partial_{\mu} A_{\alpha}-\partial_{\alpha} A_{\mu} .
$$

The lowest order correction to the graviton-photon vacuum loop will have the form (in Euclidean momentum space):

$$
\Pi_{\mu \nu \rho \sigma}^{\mathrm{Gvac}}(p)=\kappa^{2} \int \frac{d^{4} q}{(2 \pi)^{4}} V_{\mu \nu \lambda \alpha}(p,-q,-q-p)
$$




$$
\times \mathcal{F}^{\gamma}\left(q^{2}\right) D_{\lambda \beta}^{\gamma}\left(q^{2}\right) V_{\rho \sigma \beta \gamma}(-p, q, p-q) \mathcal{F}^{\gamma}\left((p-q)^{2}\right) D_{\alpha \gamma}^{\gamma}\left((p-q)^{2}\right) \mathcal{G}^{\mathrm{Gvac}}\left(q^{2}\right),
$$

where $V_{\mu \nu \rho \sigma}$ is the photon-photon-graviton vertex and in a fixed gauge:

$$
D_{\mu \nu}^{\gamma}=-\frac{\delta_{\mu \nu}}{q^{2}}
$$

is the free photon propagator. Additional contributions to $\Pi_{\mu \nu \rho \sigma}^{\mathrm{Gvac}}$ come from tadpole graphs [7].

This leads to the vacuum polarization tensor

$$
\begin{gathered}
\Pi_{\mu \nu \rho \sigma}^{\mathrm{Gvac}}(p)=\kappa^{2} \int \frac{d^{4} q}{(2 \pi)^{4}} \frac{1}{q^{2}\left[(q-p)^{2}\right]} \\
\times K_{\mu \nu \rho \sigma}(p, q) \exp \left\{-q^{2} / \Lambda_{M}^{2}-\left[(q-p)^{2}\right] / \Lambda_{M}^{2}-q^{2} / \Lambda_{\text {Gvac }}^{2}\right\} .
\end{gathered}
$$

For $\Lambda_{\text {Gvac }} \ll \Lambda_{M}$, we observe that from power counting of the momenta in the loop integral, we get

$$
\begin{gathered}
\Pi_{\mu \nu \rho \sigma}^{\mathrm{Gvac}}(p) \sim \kappa^{2} \Lambda_{\mathrm{Gvac}}^{4} N_{\mu \nu \rho \sigma}\left(p^{2}\right) \\
\sim \frac{\Lambda_{\mathrm{Gvac}}^{4}}{M_{\mathrm{PL}}^{2}} N_{\mu \nu \rho \sigma}\left(p^{2}\right)
\end{gathered}
$$

where $N\left(p^{2}\right)$ is a finite remaining part of $\Pi^{\mathrm{Gvac}}(p)$ and $M_{\mathrm{PL}} \sim 10^{19} \mathrm{GeV}$ is the Planck mass.

We now have

$$
\rho_{\mathrm{vac}} \sim M_{\mathrm{PL}}^{2} \Pi^{\mathrm{Gvac}}(p) \sim \Lambda_{\mathrm{Gvac}}^{4} .
$$

If we choose $\Lambda_{\mathrm{Gvac}} \leq 10^{-3} \mathrm{eV}$, then the quantum correction to the bare cosmological constant $\lambda_{0}$ is suppressed sufficiently to satisfy the observational bound on $\lambda$, and it is protected from large unstable radiative corrections.

This provides a solution to the cosmological constant problem at the energy level of the standard model and possible higher energy extensions of the standard model. The universal fixed gravitational scale $\Lambda_{\text {Gvac }}$ corresponds to the fundamental length $\ell_{\text {Gvac }} \leq 1 \mathrm{~mm}$ at which virtual gravitational radiative corrections to the vacuum energy are cut off.

The gravitational form factor $\mathcal{G}$, when coupled to non-vacuum SM gauge boson or matter loops, will have the form in Euclidean momentum space

$$
\mathcal{G}^{\mathrm{GM}}\left(q^{2}\right)=\exp \left[-q^{2} / \Lambda_{G M}^{2}\right]
$$


If we choose $\Lambda_{G M}=\Lambda_{M}>1-10 \mathrm{TeV}$, then we will reproduce the standard model experimental results, including the running of the standard model coupling constants, and $\mathcal{G}^{G M}\left(q^{2}\right)=\mathcal{F}^{M}\left(q^{2}\right)$ becomes $\mathcal{G}^{G M}(0)=\mathcal{F}^{M}\left(q^{2}=\right.$ $\left.m^{2}\right)=1$ on the mass shell. This solution to the CCP leads to a violation of the WEP for coupling of gravitons to vacuum energy and matter. This could be checked experimentally in a satellite Eötvös experiment on the Casimir vacuum energy [8].

We observe that the required suppression of the vacuum diagram loop contribution to the cosmological constant, associated with the vacuum energy momentum tensor at lowest order, demands a low gravitational energy scale $\Lambda_{\text {Gvac }} \leq 10^{-3} \mathrm{eV}$, which controls the coupling of gravitons to pure vacuum graviton and matter fluctuation loops.

In our finite, perturbative quantum gravity theory nonlocal gravity produces a long-distance infrared cut-off of the vacuum energy density through the low energy scale $\Lambda_{\text {Gvac }}<10^{-3} \mathrm{eV}$ [6] matter tree graphs and matter loops are controlled by the energy scale: $\Lambda_{G M}=\Lambda_{M}>1-20 \mathrm{TeV}$

The rule is: When external graviton lines are removed from a matter loop, leaving behind pure matter fluctuation vacuum loops, then those initial graviton-vacuum loops are suppressed by the form factor $\mathcal{G}^{\text {Gvac }}\left(q^{2}\right)$ where $q$ is the internal matter loop momentum and $\mathcal{G}^{\text {Gvac }}\left(q^{2}\right)$ is controlled by $\Lambda_{\text {Gvac }} \leq$ $10^{-3} \mathrm{eV}$. On the other hand, e.g. the proton first-order self-energy graph, coupled to a graviton is controlled by $\Lambda_{G M}=\Lambda_{M}>1-20 \mathrm{TeV}$ and does not lead to a measurable violation of the equivalence principle.

The scales $\Lambda_{M}$ and $\Lambda_{\text {Gvac }}$ are determined in loop diagrams by the quantum non-localizable nature of the gravitons and standard model particles. The gravitons coupled to matter and matter loops have a nonlocal scale at $\Lambda_{G M}=$ $\Lambda_{M}>1-20 \mathrm{TeV}$ or a length scale $\ell_{M}<10^{-16} \mathrm{~cm}$, whereas the gravitons coupled to pure vacuum energy are localizable up to an energy scale $\Lambda_{\text {Gvac }} \sim$ $10^{-3} \mathrm{eV}$ or down to a length scale $\ell_{\text {Gvac }}>1 \mathrm{~mm}$.

The fundamental energy scales $\Lambda_{\text {Gvac }}$ and $\Lambda_{G M}=\Lambda_{M}$ are determined by the underlying physical nature of the particles and fields and do not correspond to arbitrary cut-offs, which destroy the gauge invariance, Lorentz invariance and unitarity of the quantum gravity theory for energies $>\Lambda_{\text {Gvac }} \sim$ $10^{-3} \mathrm{eV}$. The underlying explanation of these physical scales must be sought

\footnotetext{
${ }^{2}$ The energy scale, $\Lambda_{G} \sim 10^{-3} \mathrm{eV}$, has also been considered by R. Sundrum and G. Dvali, G. Gabadadze and M. Shifman [9].
} 
in a more fundamental theory

\section{Conclusions}

We have described a possible solution to the cosmological constant problem. The particle physics resolution requires that we construct a nonlocal quantum gravity theory, which has vertex form factors that are different for gravitons coupled to quantum vacuum fluctuations and matter. This predicts a measurable violation of the WEP for coupling to vacuum energy, but not to matter-graviton couplings or to non-vacuum matter loops. This leads to a suppression of all standard model vacuum loop contributions and, thereby, avoids a fine-tuning cancellation between the "bare" cosmological constant $\lambda_{0}$ and the vacuum contribution $\lambda_{\text {vac }}$. It retains the experimental agreement of the standard model and classical Einstein gravity. A satellite Eötvös experiment for Casimir vacuum energy could experimentally decide whether nature does allow a vacuum energy WEP violation.

Even though we can succeed in our nonlocal quantum gravity scenario to explain why $\lambda_{\text {eff }}$ is small, without excessive fine tuning, we are still confronted with the "coincidence" problem associated with dark energy and the existence of a small, positive cosmological constant [1].

As a model of a future fundamental, nonlocal quantum gravity theory, it does provide clues as to the resolution of the "infamous" cosmological constant problem.

\section{Acknowledgments}

I thank Michael Clayton and George Gillies for helpful and stimulating discussions. This work was supported by the Natural Sciences and Engineering Research Council of Canada.

\section{References}

[1] Straumann, N., On the Cosmological Constant Problem and the Astronomical Evidence for a Homogeneous Energy Density with Negative

\footnotetext{
${ }^{3}$ It is interesting to note that if we choose $\Lambda_{\mathrm{GM}}=\Lambda_{M}=5 \mathrm{TeV}$, then we obtain $\Lambda_{\mathrm{Gvac}}=\Lambda_{M}^{2} / M_{\mathrm{PL}}=2.1 \times 10^{-3} \mathrm{eV}$.
} 
Pressure, astro-ph/0203330; Peebles, P. J. E., and Ratra, B., The Cosmological Constant and Dark Energy, astro-ph/0207347; Ellwanger, U., The Cosmological Constant, hep-ph/0203252.

[2] Perlmutter, S., et al. Ap. J. 483, 565 (1997), astro-ph/9608192: Riess, A. G., et al. Astron. J. 116, 1009 (1998), astro-ph/9805201.

[3] Weinberg, S., Rev. Mod. Phys. 61, 1 (1989).

[4] Cline, J., hep-ph/0207155.

[5] Moffat, J. W., Phys. Rev. D41, 1177 (1990); Evens, D., Moffat, J. W., Kleppe, G., and Woodard, R. P., Phys. Rev. D43, 499 (1991).

[6] Moffat, J. W., hep-ph/0102088 v2.

[7] Capper, D. M., Leibbrandt, G., Medrano, M. R., Phys. Rev. D8, 4320 (1973); Capper, D. M., Duff, M. J., and Halpern, L., Phys. Rev. 10, 461 (1974).

[8] Ross, D. K., Il Nuovo Cim. 114, 1073 (1999).

[9] Sundrum, R., JHEP 9907, 001 (1999), hep-ph/9708329 v2 and Dvali, G., Gabadadze, G., and Shifman, M., hep-th/0202174. 